\title{
Complex Source Radiation in a Cylindrical Radome of Metal-Dielectric Grating
}

\author{
Ayhan Altıntaş, Senior Member, IEEE, Slim Ouardani, and Vladimir B. Yurchenko
}

\begin{abstract}
The radiation fields of a line source enclosed in a circular dielectric radome with grating consisting of an array of thin lossy metal strips are analyzed. The variations of the directivity of the source beam with respect to the beam direction are studied. The possibility of damping these variations by an appropriate design of the radome is demonstrated.
\end{abstract}

Index Terms-Complex source, gratings.

\section{INTRODUCTION}

$\mathbf{T}$ WHE effect of a dielectric layer on the penetration of electromagnetic waves has been extensively analyzed in recent years due to its numerous applications such as in the design of a radar antenna enclosed in a radome [1]. Both accurate and approximate approaches have been used to study the effect and various configurations of the radome are considered [1]-[5]. Two-dimensional (2-D) configurations are of particular interest providing the most accurate analysis of the problem. In [4] and [5], full-wave analysis of two problems involving circular cylindrical radome is provided. The incident and scattered fields are presented by cylindrical functions and exact boundary conditions are applied to the fields at the radome to obtain accurate solutions.

If the wall of the radome can be modeled as a thin structure described in terms of effective boundary conditions, the computational problem is much less intense. In [6], effective boundary conditions of a new generalized form are obtained for conductive and dielectric layers as well as for multilayer compositions of different kind. They provide a useful approximation valid for the broad class of problems including the radome problems as well. Radomes of complicated structure and of special shape are particularly suitable for the approximate analysis of this kind.

In this paper, we study the effect of a circular cylindrical radome consisting of an array of thin metal strips parallel to the axis (metal grating) or, similarly, of alternating metal strips and dielectric shells that form a cylindrical surface (metaldielectric grating) on the radiation of a complex line source of $E$ polarization enclosed in the radome.

Dielectric radomes with metal supporting elements are often used in practice when reinforcement of a thin dielectric wall

Manuscript received March 11, 1998; revised June 3, 1999.

A. Altıntaş and S. Ouardani are with the Department of Electrical and Electronics Engineering, Bilkent University, Bilkent, Ankara, 06533 Turkey.

V. B. Yurchenko is with the Institute of Radiophysics and Electronics, National Academy of Sciences of Ukraine, and with the Department of Electrical and Electronics Engineering, Bilkent University, Bilkent, Ankara, 06533 Turkey.

Publisher Item Identifier S 0018-926X(99)07957-0. of the radome is needed. However, metal reinforcements are rather strong scatterers of electromagnetic waves. They degrade the antenna parameters, distort the antenna beam and decrease the directivity. Therefore, one has to design the structure carefully in order to minimize the effect of metal elements. Although actual radomes are usually of spherical shape, cylindrical structures are more feasible for the analysis providing valuable physical insight and practical recommendations valid for more complicated structures as well.

The aim of our analysis is to minimize the negative effect of the metal grating of the radome on the directivity of the antenna beam. In distinction from typical research concerning plane wave scattering by planar gratings (see, e.g., [8]), we consider the propagation of the beam through the curved metal-dielectric grating representing the wall of a cylindrical radome. We focused on studying the variations of the directivity with respect to the beam direction and found the possibility of damping these variations by the proper design of the metal-dielectric radome.

According to the geometry of our problem, the fields on the concave and convex sides of the radome are represented by modal cylindrical waves. Solution is then found by application of the boundary conditions suggested in [6] to relate the outer fields of the radome to the inner ones. Although these boundary conditions were initially derived for planar sheets, the numerical data obtained justify strongly the validity of our method for circular geometry as well. The results for the far-field patterns and the directivity are calculated for various structures as functions of the beam orientation, strip width, and the number of strips for both metal and metaldielectric gratings. Also, the dependence of the directivity on the thickness of the dielectric shell is investigated.

The organization of this paper is as follows. In Section II we introduce the basic concept of the method and the formulation of the problem. Numerical results are presented in Section III. Main conclusions follow in Section IV.

Throughout the analysis, the time-dependence $e^{-i \omega t}$ is assumed and suppressed.

\section{FORMULATION}

\section{A. Geometry of the Problem and the Radiation Source}

In this analysis, a line source with complex position is considered to simulate directed beam fields [9], [10]. Both the incident and scattered fields are represented as series of cylindrical functions and then the effect of transmission through the radome is analyzed to evaluate the total radiation 


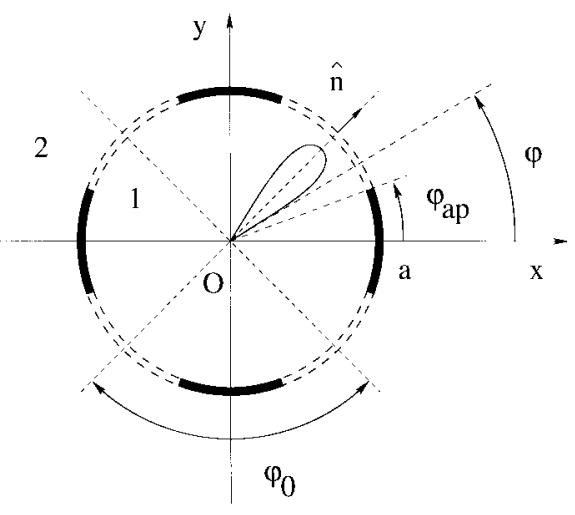

Fig. 1. Geometry of a circular metal grating. Metal strips are shown by solid arcs. Dashed arcs represent either air slots or dielectric shells depending on the problem considered.

fields. Formulation of the problem is carried out for a thin periodic metal grating in free-space and then extended to the case of periodic metal-dielectric gratings.

Fig. 1 shows a grating consisting of an array of thin metal strips of angular width $2 \varphi_{a p}$ located on a circular cylinder of radius $a$. The array is periodic with the period $\varphi_{0}=2 \pi / M$ where $M$ is the number of strips. In the case of metaldielectric gratings, the slots between metal strips are filled with dielectric shells as shown by the dashed arcs. Typical values of the parameters are given in the captions of Figs. 2-8. The radome layer is represented by a sheet with effective boundary conditions at the radius $a$.

The position of a line source parallel to $z$ axis and radiating the beam pattern in the $x, y$ plane is specified by the complex vector [9]-[11]

$$
\vec{r}_{s}=\vec{r}_{0}+i \vec{b}=r_{0} \hat{x}+i b(\cos \beta \hat{x}+\sin \beta \hat{y})
$$

where $i=\sqrt{-1}$, the real number $b$ is a measure of the source directivity, and the angle $\beta$ represents the beam direction.

The incident electric field due to the line source at the complex position $\vec{r}_{s}$ is given by

$$
E_{z}^{i n}(\vec{r})=H_{0}^{(1)}\left(k\left|\vec{r}-\vec{r}_{s}\right|\right)
$$

where $k$ is the free-space wavenumber and $H_{0}^{(1)}(\cdot)$ is the Hankel function of the first kind. With the use of the addition theorem for the Hankel function, $E_{z}^{i n}(\vec{r})$ can be written as [11]

$$
E_{z}^{i n}(r, \varphi)=\sum_{n} s_{n} H_{n}^{(1)}(k r) e^{i n \varphi}, \quad r>\left|r_{s}\right|
$$

where $s_{n}=J_{n}\left(k r_{s}\right) e^{-i n \varphi_{s}}, J_{n}(\cdot)$ are the Bessel functions, $r_{s}=\sqrt{r_{0}^{2}-b^{2}+2 i b r_{0} \cos \beta}$, Re $r_{s}>0$, and $\varphi_{s}=$ $\cos ^{-1}\left(\left(r_{0}+i b \cos \beta\right) / r_{s}\right)$. If $b \neq 0$, the values of $r_{s}$ and $\varphi_{s}$ are complex numbers that results in the beam pattern of the complex source as shown by dotted curves in Fig. 8.

\section{B. Boundary Conditions}

The total field $E_{z}(\vec{r})$ satisfies 2-D Helmholtz equation

$$
\left(\frac{\partial^{2}}{\partial r^{2}}+\frac{1}{r} \frac{\partial}{\partial r}+\frac{1}{r^{2}} \frac{\partial^{2}}{\partial \varphi^{2}}+k^{2}\right) E_{z}(\vec{r})=0
$$

subject to the boundary conditions at $r=a$ and the Sommerfeld radiation condition at infinity.

The boundary conditions we use are given in the form [6]

$$
\begin{aligned}
\frac{1}{2}\left[\vec{E}_{T 2}(\varphi)+\vec{E}_{T 1}(\varphi)\right] & =R(\varphi) \hat{n} \times\left[\vec{H}_{T 2}(\varphi)-\vec{H}_{T 1}(\varphi)\right] \\
\frac{1}{2}\left[\vec{H}_{T 2}(\varphi)+\vec{H}_{T 1}(\varphi)\right] & =-S(\varphi) \hat{n} \times\left[\vec{E}_{T 2}(\varphi)-\vec{E}_{T 1}(\varphi)\right]
\end{aligned}
$$

where $\vec{E}_{T 1,2}(\varphi)$ and $\vec{H}_{T 1,2}(\varphi)$ are the tangential components of electric and magnetic fields at the interface when $r \rightarrow a \mp 0$, respectively, $R(\varphi)$ and $S(\varphi)$ are the parameters interpreted as the electric resistivity and magnetic conductivity of the interface, respectively, and $\hat{n}$ is the outward unit normal.

In the case of $E$ polarization, the boundary conditions (5) and (6) relate the fields $E_{z}$ and $H_{\varphi}$ in the following way:

$$
\begin{aligned}
& H_{\varphi 2}(\varphi)-H_{\varphi 1}(\varphi)=i Z_{0}^{-1} \xi(\varphi)\left[E_{z 2}(\varphi)+E_{z 1}(\varphi)\right] \\
& E_{z 2}(\varphi)-E_{z 1}(\varphi)=-i Z_{0} \eta(\varphi)\left[H_{\varphi 2}(\varphi)+H_{\varphi 1}(\varphi)\right]
\end{aligned}
$$

where

$$
\xi(\varphi)=-\frac{i}{2} \frac{Z_{0}}{R(\varphi)}, \quad \eta(\varphi)=\frac{i}{2} \frac{1}{Z_{0} S(\varphi)}
$$

and $Z_{0}$ is the intrinsic impedance.

Notice that the boundary conditions of this kind, although written in another form, were derived earlier for absorbing shells in [12] (with extension for the curved surfaces), for cylindrical metal membranes in [13], and for electric and magnetic current sheets in [14] (the sheet model [14] relates (6) and (8) to magnetic sheets only, providing no general formulas for $R$ and $S$ ).

The electric resistivity $R$ and the magnetic conductivity $S$ are complex parameters which depend on the material properties and thickness of the interface layer. For a relatively thin layer $(k h \ll 1)$ characterized by material parameters $\epsilon, \mu, \sigma$ and thickness $h$, under the condition of $|Z| \ll Z_{0}$ where $Z=Z_{0} / \sqrt{\tilde{\epsilon}_{r}}$ is the impedance of the interface medium $\left(\tilde{\epsilon}_{r}=\epsilon_{r}+i \sigma / \omega \epsilon_{0}, \epsilon_{r}=\epsilon / \epsilon_{0}, \mu=\mu_{0}\right)$, the respective values of $R$ and $S$ are given as [6]

$$
R=\frac{i}{2} Z \cot \left(\frac{k_{\epsilon} h}{2}\right) \quad \text { and } S=\frac{i}{2} \frac{1}{Z} \cos \left(\frac{k_{\epsilon} h}{2}\right)
$$

where $k_{\epsilon}=k \sqrt{\tilde{\epsilon}_{r}}$ (one can easily reproduce (10) by considering a plane wave normally incident on a slab). Note that $|Z| \ll Z_{0}$ assumes $\left|\tilde{\epsilon}_{r}\right| \gg 1$. Substituting (10) in (9), one gets

$$
\xi=-\sqrt{\tilde{\epsilon}_{r}} \tan \left(\frac{k_{\epsilon} h}{2}\right) \text { and } \eta=\frac{1}{\sqrt{\tilde{\epsilon}_{r}}} \tan \left(\frac{k_{\epsilon} h}{2}\right)
$$

that implies

$$
\eta=-\xi / \tilde{\epsilon}_{r}, \quad|\eta| \ll|\xi| .
$$

Relations (12) in conjunction with (7) and (8) justify under the conditions $|\xi| \ll\left|\tilde{\epsilon}_{r}\right|$ and $\left|\tilde{\epsilon}_{r}\right| \gg 1$, the validity (except very oblique wave incidence [15]) of the resistivity approximation $\eta=0$ which is widely used for the analysis of scattering from conducting and dielectric shells [15]-[17] (the approximation breaks if $\left.\left|\tan \left(k_{\epsilon} h / 2\right)\right| \gg\left|\tilde{\epsilon}_{r}\right|^{1 / 2}\right)$. 
Depending on the values of $h$ and $\tilde{\epsilon}_{r}$, there are four limiting cases of interest: a very thin or relatively thick layer of either metal or dielectric material.

In the case of a thin layer characterized by inequality $\left|k_{\epsilon} h\right| \ll 1$ (penetrable sheet), relations (10) and (11) yield $R \approx 1 / \sigma h$ [14] and $\xi \approx-i Z_{0} \sigma h / 2$ for metal strips $\left(\tilde{\epsilon}_{r} \approx\right.$ $\left.i \sigma / \omega \epsilon_{0}=i \sigma Z_{0} / k\right), R \approx i Z_{0} / \epsilon_{r} k h[18]$ and $\xi \approx-\epsilon_{r} k h / 2$ for dielectric shells $\left(\tilde{\epsilon}_{r} \approx \epsilon_{r} \gg 1\right)$, and $S \approx i / Z_{0} k h$ and $\eta \approx k h / 2$ for both of them. In all the cases, one has $|R| \gg R_{\min }$ and $|S| \gg R_{\min }^{-1}$ where $R_{\min }=|Z| \ll Z_{0}$ that results in $|\xi| \ll\left|\tilde{\epsilon}_{r}\right|^{1 / 2}$ and $|\eta| \ll\left|\tilde{\epsilon}_{r}\right|^{-1 / 2} \ll 1$. The latter means that the resistivity approximation $\eta=0$ is always justified for a thin layer with $\left|\tilde{\epsilon}_{r}\right| \gg 1$.

In the case of a relatively thick layer, $\left|k_{\epsilon} h\right| \gg 1$, one has $R \approx Z / 2=(1-i) / 2 \sigma \delta, \xi \approx-i Z_{0} / Z=(1-i) Z_{0} \sigma \delta / 2$, $S \approx 1 / 2 Z=(1+i) \sigma \delta / 4$, and $\eta \approx i Z / Z_{0}=(1+i) / Z_{0} \sigma \delta$ for metal strips (impenetrable screens, $\delta \ll h, \delta=\sqrt{2 / \sigma \omega \mu_{0}}$ is the skin depth, $|Z| \ll Z_{0}$, the approximation $\eta=0$ also valid), and the generic relations (10) and (11) for dielectric shells with real values $\tilde{\epsilon}_{r}=\epsilon_{r} \gg 1$ being assumed.

Notice that, for the impenetrable screen, the resistivity $R$ is $0.5 Z$ but not $Z$, the value of $R$ is determined by $\delta \ll h$ instead of $h$, and Leontovich impedance boundary conditions [19] take place. Notice also that neither of $|R|=Z_{0},|2 R|=Z_{0}$, $|S|=1 / Z_{0}$ or $|2 S|=1 / Z_{0}$ represents a free-space layer. Instead, it is $h=n \lambda_{\epsilon}=n \lambda / \sqrt{\tilde{\epsilon}_{r}}(\lambda$ is the free-space wavelength, $\tilde{\epsilon}_{r}$ is assumed real, $\left.n=0,1,2, \cdots\right)$ that simulates a free-space interface providing $\xi=\eta=0$ (the case of $\epsilon_{r}=1$ and $\sigma=0$ is inappropriate because of assumption $\left|\tilde{\epsilon}_{r}\right| \gg 1$ ).

Of a special interest is a perfect dielectric of thickness $h=(2 n-1) \lambda_{\epsilon} / 2$. In this case, both $|\xi|$ and $\mid \eta$ tend to infinity (there is no resistivity approximation) providing the boundary conditions

$$
E_{z 2}(\varphi)=-E_{z 1}(\varphi) \quad \text { and } \quad H_{\varphi 2}(\varphi)=-H_{\varphi 1}(\varphi)
$$

The conditions mean the inversion of the field phase across the boundary, with no change of the field amplitude.

The similar effect, although related to the electric field only, happens at the metal strips when $\sigma \rightarrow \infty$ while $h=$ const (perfectly conducting screens). In this case, $|\xi| \rightarrow \infty, \eta \rightarrow 0$, and the boundary conditions are

$$
E_{z 2}(\varphi)=-E_{z 1}(\varphi) \quad \text { and } \quad E_{z 2}(\varphi)=E_{z 1}(\varphi)
$$

providing eventually $E_{z 2}(\varphi)=E_{z 1}(\varphi)=0$ (notice that the apparent conflict of "impenetrability" and "transparency" of a thin screen is only an ostensible one since the electric field is continuous across the screen not because of "transparency" but due to high conductivity providing $E_{z}=0$ at the surface; see also [20] for a relevant discussion).

A remarkable similarity of the field behavior in the two cases arising due to similarity of the boundary conditions (13) and (14) is the physical basis for damping the angular variations of the directivity of the radiated beam considered in Section III.

\section{Solution Technique}

Coming back to our problem, we note that metal strips form a periodic contour. Thus, the parameters $\xi$ and $\eta$ are periodic step functions of $\varphi$. For a metal grating in free-space, the coefficients $\xi$ and $\eta$ take the values $\xi_{M}$ and $\eta_{M}$ at the metal strips and drop to zero at the slots $\left(\xi_{S}=\eta_{S}=0\right)$ to account for the continuity of the total electric and magnetic fields there. If the slots are filled with a dielectric material (the case of metal-dielectric grating), $\xi$ and $\eta$ do not vanish between the metal strips but take the values $\xi_{S}$ and $\eta_{S}$ calculated from (11) corresponding to the properties of the dielectric shells inserted.

Further, we assume nonperfectly conducting metal strips $\left(\left|\xi_{M}\right| \neq \infty\right)$ and use the direct method for solving (4) in a manner similar to [8]. Due to the axial symmetry of the problem, the field $E_{z}(\vec{r})$ is expanded in a series as

$$
\begin{aligned}
E_{z}(\vec{r})= & \sum_{n=-\infty}^{\infty} e^{i n \varphi} \\
& \cdot \begin{cases}r_{n} J_{n}(k r)+s_{n} H_{n}^{(1)}(k r), & \left|r_{s}\right|<r<a \\
t_{n} H_{n}^{(1)}(k r), & r>a\end{cases}
\end{aligned}
$$

where $r_{n}$ and $t_{n}$ are the unknown coefficients to be determined by the boundary conditions at $r=a$. When $t_{n}$ found, we can compute the far-field pattern $U(\varphi)$ and the beam directivity $D_{\beta}$ as

$$
U(\varphi)=\sum_{n=-\infty}^{\infty} t_{n} i^{-n} e^{i n \varphi}
$$

and

$$
D_{\beta}=|U(\beta)|^{2} / \sum_{n=-\infty}^{\infty}\left|t_{n}\right|^{2} .
$$

To solve for $r_{n}$ and $t_{n}$, we expand the functions $\xi(\varphi)$ and $\eta(\varphi)$ in their Fourier series and substitute them in (7) and (8) together with the expansions (15). In this way, the following are obtained:

$$
\begin{gathered}
t_{m} H_{m}^{\prime}-r_{m} J_{m}^{\prime}-\xi_{0} \sum_{n=-\infty}^{\infty} f_{m-n}\left(t_{n} H_{n}+r_{n} J_{n}\right) \\
=s_{m} H_{m}^{\prime}+\xi_{0} \sum_{n=-\infty}^{\infty} f_{m-n} s_{n} H_{n} \\
t_{m} H_{m}-r_{m} J_{m}-\eta_{0} \sum_{n=-\infty}^{\infty} g_{m-n}\left(t_{n} H_{n}^{\prime}+r_{n} J_{n}^{\prime}\right) \\
=s_{m} H_{m}+\eta_{0} \sum_{n=-\infty}^{\infty} g_{m-n} s_{n} H_{n}^{\prime}
\end{gathered}
$$

where $H_{n}=H_{n}^{(1)}(k a), H_{n}^{\prime}=H_{n}^{(1) \prime}(k a), J_{n}=J_{n}(k a)$, $J_{n}^{\prime}=J_{n}^{\prime}(k a)$, prime denotes the derivatives with respect to the argument, $\xi_{0}=\xi_{M}-\xi_{S}, \eta_{0}=\eta_{M}-\eta_{S}, f_{n}=$ $g_{n}=\psi \sin \left(n M \varphi_{a p}\right) /\left(n M \varphi_{a p}\right)$ if $n \neq 0, f_{0}=\psi+\xi_{S} / \xi_{0}$, $g_{0}=\psi+\eta_{S} / \eta_{0}, \psi=M \varphi_{\text {ap }} / \pi$, and $m, n=0, \pm 1, \pm 2, \cdots$. 
Substituting $x_{n}=\left(t_{n}+s_{n}\right) H_{n}^{\prime}$ and $y_{n}=r_{n} H_{n}$, we reduce (18) and (19) to the form

$$
\begin{aligned}
\xi_{0} & \sum_{n=-\infty}^{\infty} f_{m-n}\left[x_{n} H_{n} / H_{n}^{\prime}+y_{n} J_{n} / H_{n}\right] \\
& -x_{m}+y_{m} J_{m}^{\prime} / H_{m}=-2 s_{m} H_{m}^{\prime}, \\
\eta_{0} & \sum_{n=-\infty}^{\infty} g_{m-n}\left[x_{n}+y_{n} J_{n}^{\prime} / H_{n}\right] \\
& \quad-x_{m} H_{m} / H_{m}^{\prime}+y_{m} J_{m} / H_{m}=-2 s_{m} H_{m} .
\end{aligned}
$$

In this form, (20) and (21) were truncated assuming $|m|,|n| \leq$ $N$ and, upon the symmetry splitting, solved numerically by LU decomposition method.

In the resistivity approximation $\eta(\varphi)=0,(20)$ and (21) are reduced to

$$
z_{m}+\sum_{n=-\infty}^{\infty} a_{m n} z_{n}=b_{m}
$$

where $z_{m}=t_{m} H_{m}(|m|+1)^{1 / 2}, a_{m n}=\alpha J_{m} H_{m} f_{m-n}[(|m|+$ 1)/(|n|+1) $]^{1 / 2}, \alpha=i \pi k a \xi_{0}$, and $b_{m}=s_{m} H_{m}(|m|+1)^{1 / 2}$.

For one metal strip, (22) is identical to (28)-(31) in [16] (with $\gamma_{p}=0$ ) obtained by the integral equation method. The identity is verified by presenting (28)-(31) of [16] in terms of the far-field coefficients $t_{n}=x_{n} J_{n}+s_{n}$ instead of the current coefficients $x_{n}$ defined in (24) of [16], that proves the equivalence of the two approaches (note that functions $f_{m-n}$ and $g_{m-n}$ in (20), (21) are of the same kind as functions $S_{m n}$ defined in [16]). Equations (16)-(19) in [17] would also coincide with (22) if the parameter $-R / 2$ is used in those equations instead of $R$, with the relevant plots being correct for the values of resistivity twice less than specified in the captions. Thus, the mode-matching technique implemented above is exactly equivalent to the method used in [16] and [17] providing the same level of reliability of solutions.

The accuracy and convergence of numerical solutions were tested for various values of $\xi_{M}, \eta_{M}, \xi_{S}$, and $\eta_{S}$, Fig. 2. As one can see, if $N>k a$, the relative accuracy of $D_{\beta}$ estimated from the convergence process for the given values of the parameters is about $1 \%$. However, since (20)-(22) are not regularized (if $R \neq 0$, regularization of these equations is not necessary [16], [17]), the accuracy of $D_{\beta}$ does not seem to improve when $N \gg k a$ (see Fig. 2) and decreases with increasing $M$ or with decreasing $|R|$. Still, the reciprocity and optical theorems for lossless structures under the plane wave excitation are satisfied exactly, as in [17], with the accuracy $10^{-16}$ independent of the truncation number even for $N<k a$. Generally, the solutions obtained are rather stable, although the convergence is worse for lossless structures as compared to lossy ones of the same value of $|R|$. Therefore, we believe, the comparison of our results with regularized solutions would be of interest, especially for structures of greater number of strips and of greater conductivity of metal, which has to be done elsewhere.

With respect to the approach we used, a comment concerning the Fourier factorization of (7)-(8) yielding (18)-(19) is worthwhile. Since all the quantities in (7)-(8) are generally discontinuous functions of $\varphi$ at the strip edges, both in metal

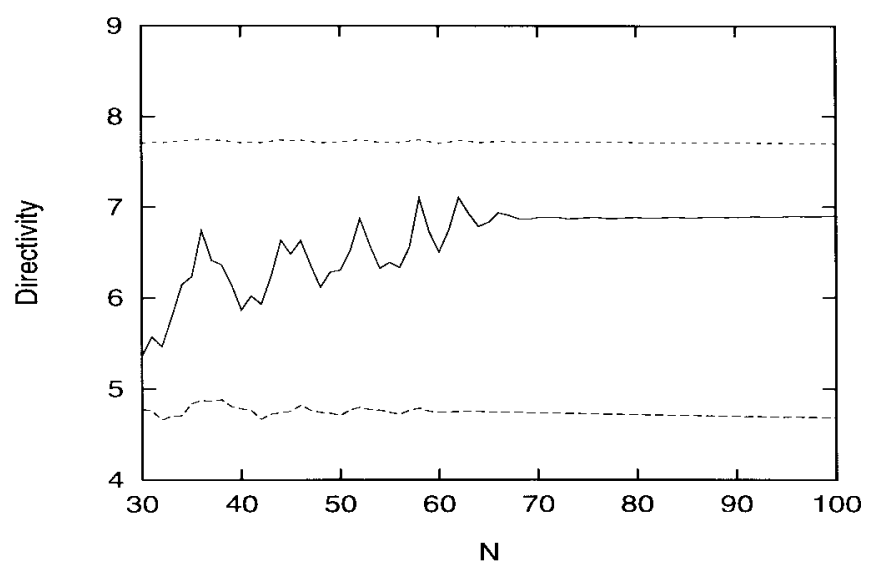

(a)

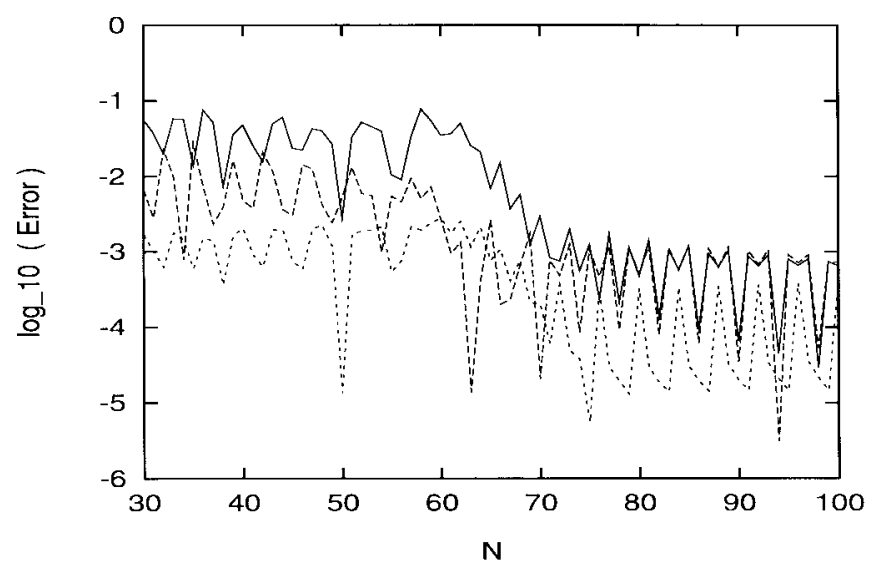

(b)

Fig. 2. (a) Directivity $D_{\beta}$ and (b) its computational error $\Delta D_{\beta}=\log _{10}\left(\left|D_{\beta}^{N}-D_{\beta}^{N+1}\right| / D_{\beta}^{N}\right)$ as functions of the truncation number $N$ for a circular grating of $M=4$ impenetrable metal strips with air slots (solid curves) and with dielectric shells characterized by $\epsilon_{r}=16$, $h=0.4 \lambda_{\epsilon}$ (dashed curves), and by $\epsilon_{r}=16+0.5 i, h=0.5 \lambda / \operatorname{Re}\left(\sqrt{\tilde{\epsilon}_{r}}\right)$ (dotted curves). Other relevant parameters are $Z / Z_{0}=0.1(1-i)$, $\varphi_{a p}=0.5^{\circ}, k a=62.8, r_{0}=0, \beta=0^{\circ}$, and $k b=5$ that corresponds to the beam halfwidth at half power $\varphi_{b}=21.4^{\circ}$ and to the source directivity in free space $D_{\beta}=7.82$.

gratings and in metal-dielectric ones, the Fourier factorization of the truncated series is a tricky mathematical operation in view of the Gibbs phenomenon, as clearly shown in [21].

The factorization we used is consistent with suggestions [21] that partially overcome the problem if discontinuities of the sums $e_{p}=E_{z 2}(\varphi)+E_{z 1}(\varphi)$ and $h_{p}=Z_{0}\left[H_{\varphi 2}(\varphi)+H_{\varphi 1}(\varphi)\right]$ in the right-hand parts of (7)-(8) are less significant than discontinuities of the differences $e_{m}=E_{z 2}(\varphi)-E_{z 1}(\varphi)$ and $h_{m}=Z_{0}\left[H_{\varphi 2}(\varphi)-H_{\varphi 1}(\varphi)\right]$ in the left-hand parts. Generally, it depends on the values and jumps of $\xi$ and $\eta$ and can be tested as in Fig. 3. The relations between the jumps of $e_{p}, h_{p}, e_{m}$, and $h_{m}$ depend mainly on the parameters of the dielectric shells. In the case of Fig. 3(b), the jumps are minimal for both $e_{p}$ and $h_{p}$ while in Fig. 3(c) the jump is negligible only for $h_{p}$. The latter represents the electric current which is continuous at the metal-dielectric junction in this particular case that decreases the edge diffraction at the metal strips.

If the opposite relation happens in (7) or (8) —or in both of them-and the relevant function $\xi(\varphi)$ or $\eta(\varphi)$ is nonzero 


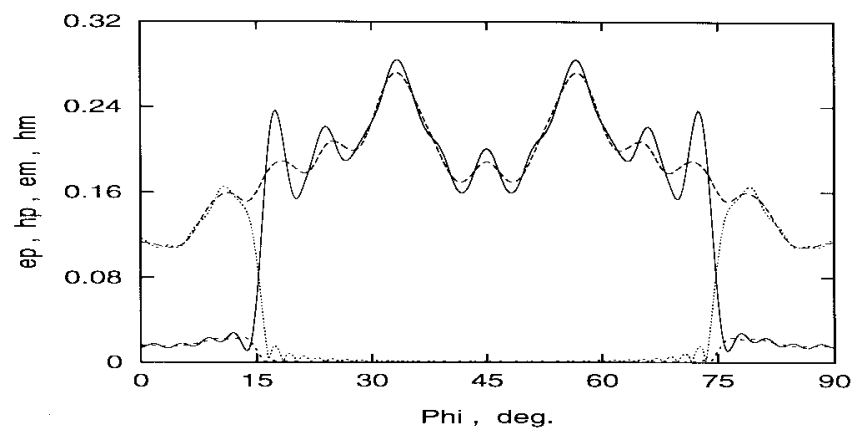

(a)

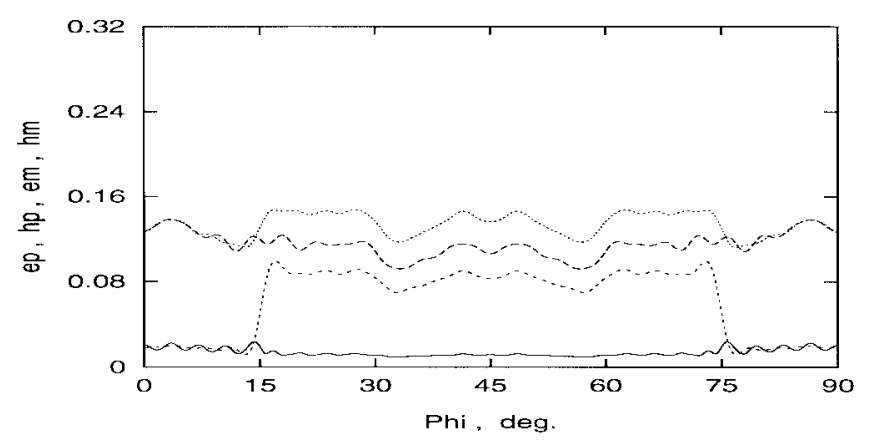

(b)

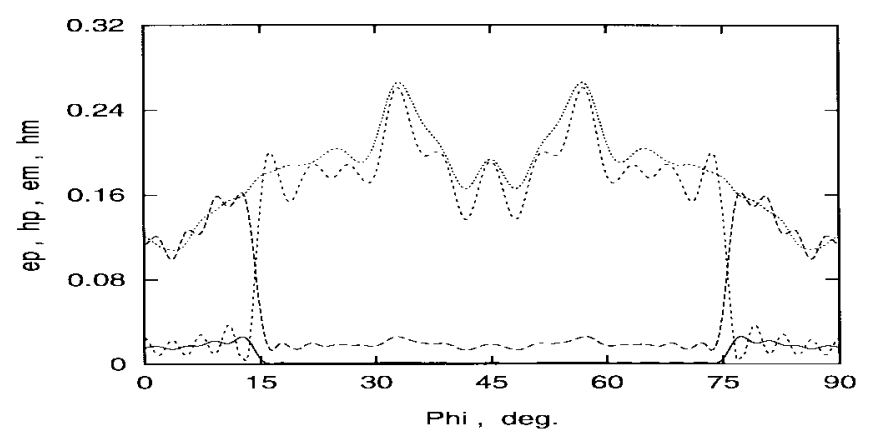

(c)

Fig. 3. Functions $e_{p}(\varphi), h_{p}(\varphi), e_{m}(\varphi)$, and $h_{m}(\varphi)$ (solid, long-dashed, short-dashed, and dotted curves, respectively) for the gratings with (a) air slots and (b) and (c) dielectric shells characterized by (b) $\epsilon_{r}=16, h=0.4 \lambda_{\epsilon}$, and (c) $\epsilon_{r}=16+0.5 i, h=0.5 \lambda / \operatorname{Re}\left(\sqrt{\tilde{\epsilon}_{r}}\right)$ in the case of $\varphi_{a p}=15^{\circ}$ and $k b=0$.

(that is possible only in a metal-dielectric grating), the relevant equation should be factorized after dividing it by $\xi(\varphi)$ or $\eta(\varphi)$ to separate the most significant discontinuities of the unknown functions and of the coefficients $\xi(\varphi), \eta(\varphi)$, or inverse ones in the opposite parts of the equation. In general, however, both the sums and differences of the fields are discontinuous functions of $\varphi$ and the method [21] does not help to overcome the problem of this kind.

\section{NUMERICAL RESULTS}

In this section, we discuss the results for the radiation patterns and the directivity obtained for various gratings of different number of strips $M$, aperture angle $\varphi_{a p}$, and beam orientation $\beta$.

For the validation of the method, we have compared the radiation patterns of a circular reflector antenna (Fig. 4) with

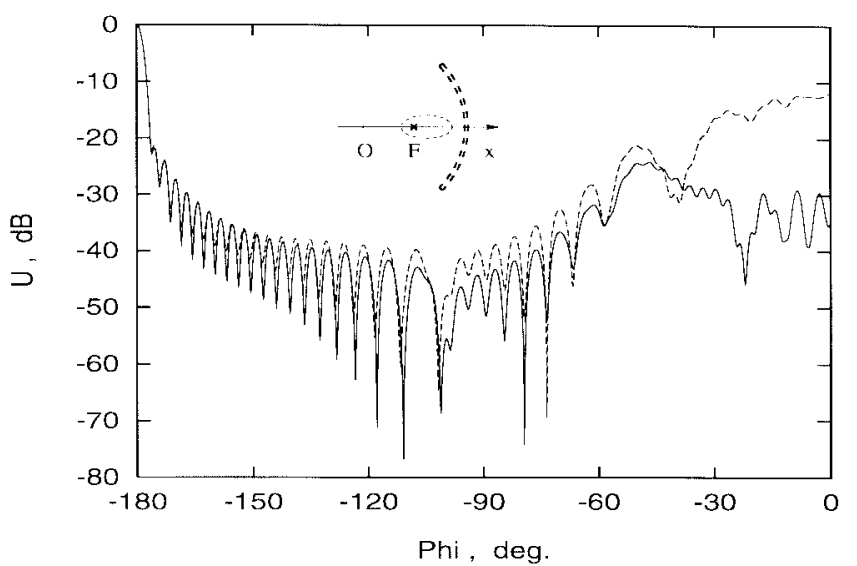

(a)

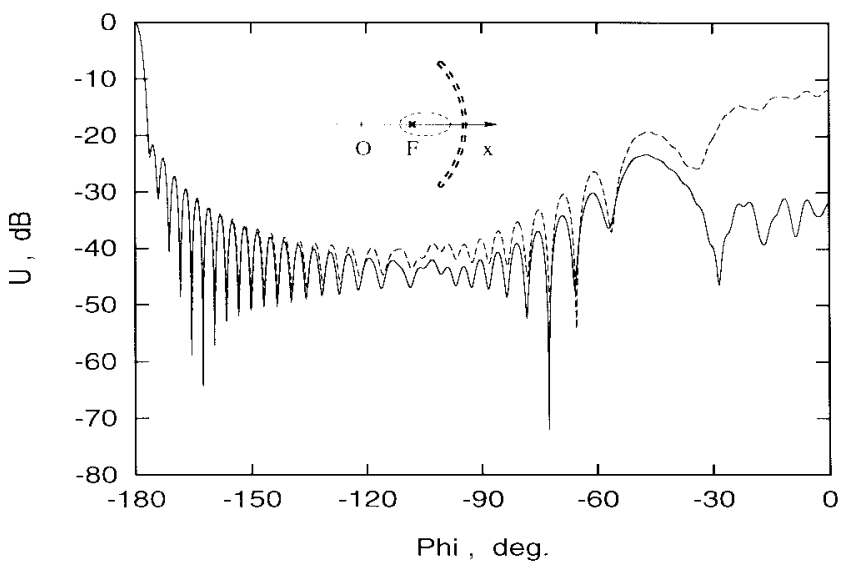

(b)

Fig. 4. Far-field pattern $U(\varphi)$ of a circular reflector antenna in the resistivity approximation $\left(S^{-1}=0\right.$, solid curve) compared to the correct solution (dashed curve) in the case of a perfect dielectric $\left(\epsilon_{r}=16\right)$ of different thickness. (a) $h=0.4 \lambda_{\epsilon}$ providing $R / Z_{0}=i 0.04$ and $S^{-1} Z_{0}^{-1}=-i 1.54$. (b) $h=0.6 \lambda_{\epsilon}$ providing $R / Z_{0}=-i 0.04$ and $S^{-1} Z_{0}^{-1}=i 1.54$ (cf. Fig. 3(b) in [16], dashed curve, where $R / Z_{0}=-i 0.1$ but $S^{-1}=0$ ). Other relevant parameters are $k a=183.7, \varphi_{a p}=20^{\circ}, r_{0}=a / 2, k b=5$, and $\beta=0^{\circ}$.

the integral equation solutions (see [16, fig. 3]), and identical results were obtained with both (20)-(21) and (22).

The results obtained for various gratings are shown in Figs. 5-8. Fig. 5 presents the variations of the overall directivity $D_{\beta}$ with angular width of metal strips for gratings of different number of strips with air slots. As expected, increasing the strip width $d$ decreases the directivity. For gratings of a relatively good conductor, a sharp drop in the directivity at small values of the aperture angle is observed. The drop is, however, smoother when strips of greater resistivity are used. This is advantageous in practice since a designer may not be restricted to extremely narrow strips.

We would like to emphasize that the above results were obtained within the resistivity approximation $\eta=0$ used for a thin dielectric layer or resistive sheet when the transparency boundary conditions are acceptable. These boundary conditions follow from (5) and (6) by setting $S=\infty$ and $R \neq 0$. The approximation looses the accuracy with decreasing magnetic conductivity $S$ that happens with increasing dielectric thickness $h$ as shown in Fig. 4. In this case, the boundary 


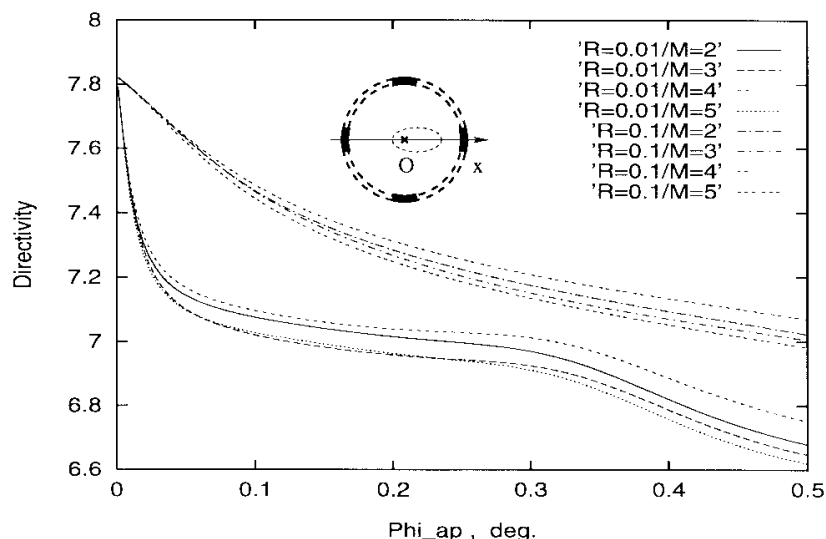

Fig. 5. Directivity $D_{\beta}$ versus aperture angle $\varphi_{a p}$ for gratings of $M=2,3,4$, and 5 metal strips in the resistivity approximation: $R / Z_{0}=0.01$ for the bottom group of curves; $R / Z_{0}=0.1$ for the upper group. Other parameters are $k a=62.8, r_{0}=0, k b=5$, and $\beta=0^{\circ}$. The angle $\varphi_{a p}=0.5^{\circ}$ corresponds to the strip width $d=0.175 \lambda$.

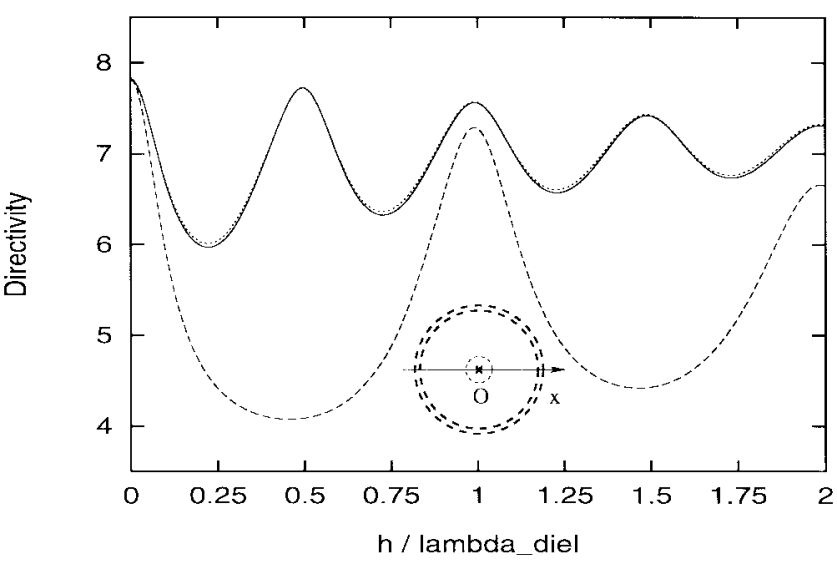

Fig. 6. Directivity $D_{\beta}$ versus thickness $h$ of the closed dielectric radome: a solution of (20) and (21) (solid curve), resistivity approximation $\eta=0$ (dashed curve), and the accurate result following [5] (dotted curve that almost coincides with the solid one). The parameters are $k a=62.8, r_{0}=0, k b=5$, $\beta=0^{\circ}$, and $\tilde{\epsilon}_{r}=4+i 0.5$. The accuracy of (20) and (21) is expected to further increase if the curvature corrections [12] are accounted.

conditions of the general form are needed, with the coefficients $R$ and $S$ as defined in (10).

To check the validity of the general boundary conditions (5) and (6) for a curved sheet, the plot of the directivity for a circular dielectric shell versus shell thickness is obtained and compared with the exact solution found by the method [5] as demonstrated in Fig. 6. As one can see, the two solutions fit pretty well.

Fig. 7 presents the directivity variations with beam direction. The directivity shows wide variations as a function of the beam orientation in the case of metal grating surrounded by vacuum. This behavior is not desired in practice. When dielectric shells are inserted between metal strips, these variations are reduced considerably as shown in Fig. 7(a). In this case, wave scattering due to metal strips is compensated by the presence of dielectric shells.

The directivity variations tend to disappear completely when perfect dielectric of half-wavelength thickness is used, in view of similarity of the boundary conditions (13) and (14)

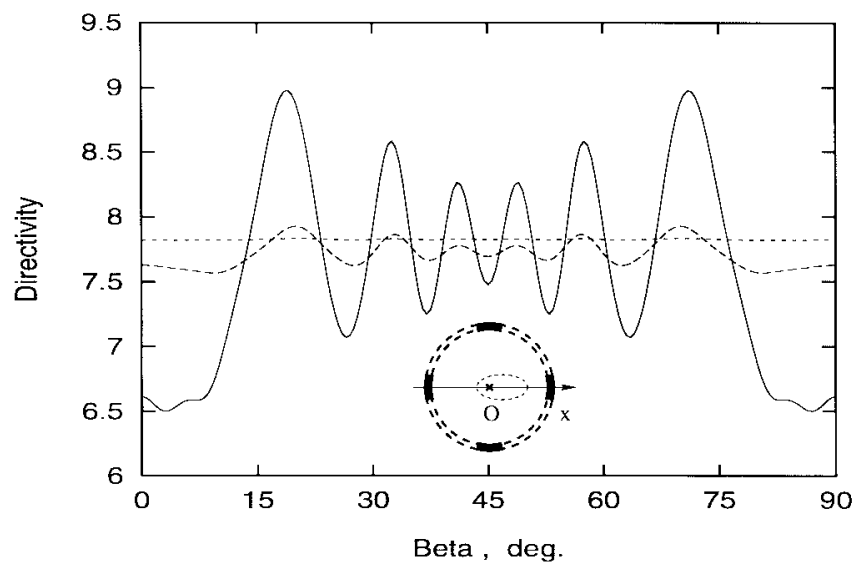

(a)

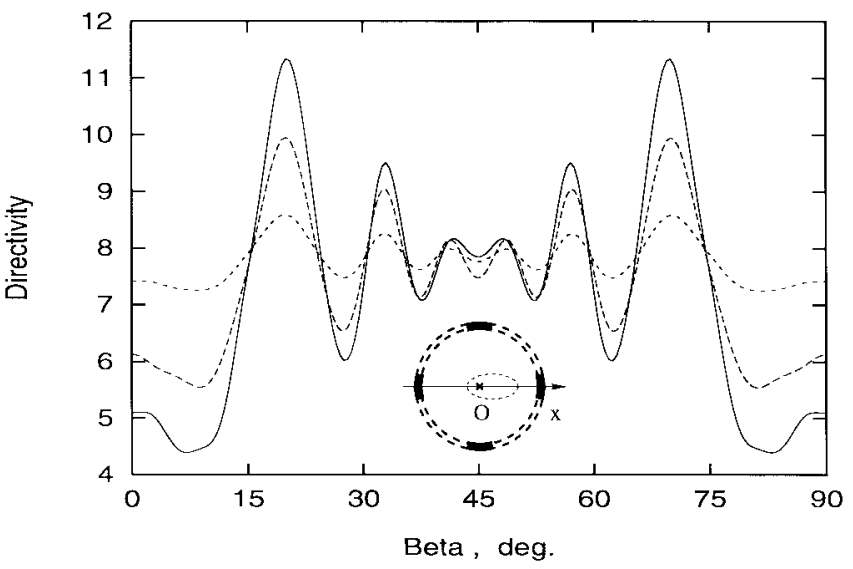

(b)

Fig. 7. Directivity $D_{\beta}$ versus beam direction $\beta$ for the metal grating (solid curve) and for the metal-dielectric ones $\left(\tilde{\epsilon}_{r}=4+i 0.5\right.$ and $\tilde{\epsilon}_{r}=4+i 0.01$, dashed and dotted curves, respectively): (a) $\varphi_{a p}=0.5^{\circ}$ and (b) $\varphi_{a p}=2^{\circ}$. The parameter $\tilde{\epsilon}_{r}$ refers to the dielectric shells of thickness $h=0.5 \lambda / \operatorname{Re}\left(\sqrt{\tilde{\epsilon}_{r}}\right)$. Impenetrable metal strips $(M=4)$ are characterized by $Z / Z_{0}=(1-i) 0.01$ that means $R / Z_{0}=(1-i) 0.005$ and $S^{-1} Z_{0}^{-1}=(1-i) 0.02$. Other parameters are $k a=62.8(a=10 \lambda)$ and $k b=5\left(\varphi_{b}=21.4^{\circ}\right)$.

discussed above. The effect is especially strong for narrow metal strips. With the strip width and the dielectric loss increasing, the directivity variations increase as well and tend to the ones for gratings in free-space as observed in Fig. 7(b). Damping the variations is, however, possible even in this case but extremely perfect dielectric of precisely half-wavelength thickness is needed. The latter is not feasible in practice. Therefore, the use of metal strips of relatively small width, as a guide $d<\lambda / 2$, is required.

The effect is illustrated by the far-field patterns shown in Fig. 8. The patterns are plotted for the gratings with air slots (short-dashed curves) and with dielectric shells of halfwavelength thickness (long-dashed and solid curves for the shells of greater and smaller losses, respectively) when the beam is directed either toward the metal strip [Fig. 8(a)] or toward the center of the slot between two strips [Fig. 8(b)]. One can see the effect of multiple scattering due to the edge diffraction in the gratings with air slots and the reduction of scattering in the structures with dielectric shells. 


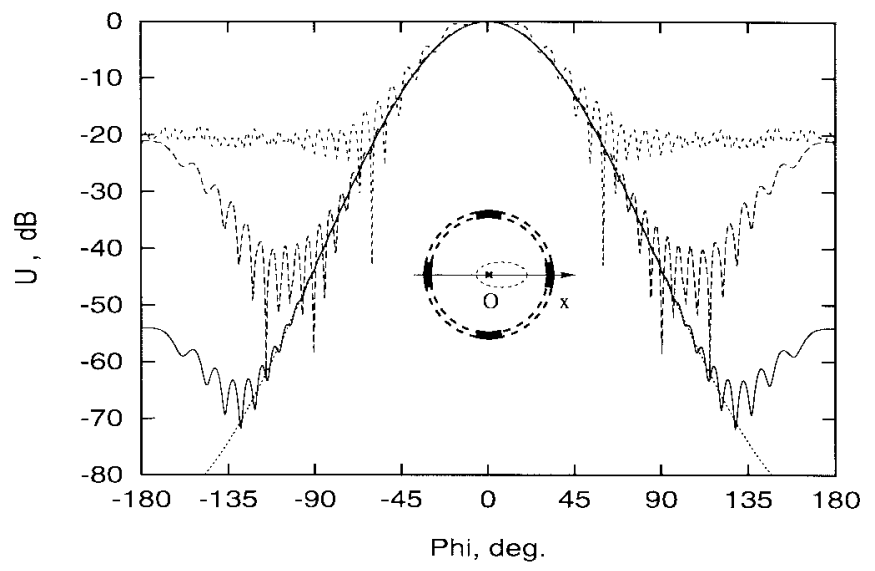

(a)

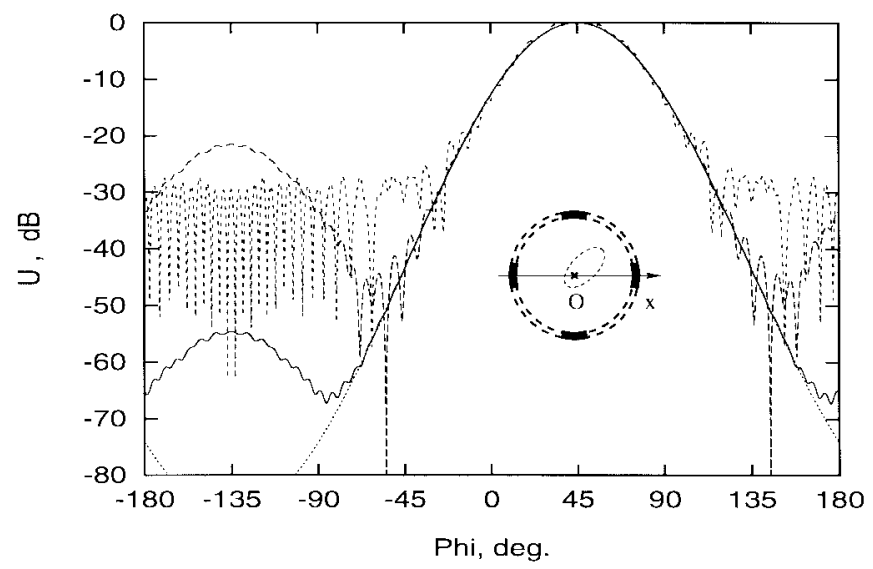

(b)

Fig. 8. Far-field patterns for the gratings with air slots (short-dashed curves) and with dielectric shells of half-wavelength thickness (long-dashed and solid curves for the shells of greater and smaller losses $\tilde{\epsilon}_{r}=4+i 0.5$ and $\tilde{\epsilon}_{r}=4+i 0.01$, respectively). The beam is directed either (a) toward the metal strip or (b) toward the center of the slot between two strips (the incident beam is shown by dotted curve). The parameters are the same as in Fig. 7 , with $\varphi_{a p}=0.5^{\circ}$.

The reason for the reduction of scattering is clearly seen by comparing the currents induced in the strips and in the dielectric shells, as shown in Fig. 3(c). It is the induction of the displacement currents in the dielectric shells that decreases the effect of discontinuity at the metal-dielectric junctions. If the displacement currents in the dielectric shells are adjusted to the conductive currents in the metal strips, the edge diffraction is minimized and the beam propagates with minimal distortion. The condition is satisfied to the best extent if the dielectric shells are perfectly lossless and precisely of half-wavelength thickness.

\section{CONCLUSION}

In this paper, we have numerically simulated the effect of a cylindrical radome that consists of a periodic array of dielectric shells and resistive metal strips on the directivity of the complex source beam radiated through the radome. By varying the parameters of metal strips and dielectric shells, we have found that the directivity can be effectively stabilized if lossless dielectric shells of precisely half-wavelength thickness are filling the slots between metal strips. The effect arises due to excitation of the displacement current in the dielectric shells adjusted to the conductive current in metal elements that decreases the edge diffraction at the metal strips.

The effect is expected to occur in three-dimensional structures as well, e.g., for radomes of spherical and more complicated shape if only the effective boundary conditions of the given kind remain applicable. Basically, it is the jump of the phase of the electric field being the same at the opposite sides of metal strips and of dielectric shells of half-wavelength thickness that minimizes the edge diffraction at the metaldielectric junctions. For this reason, the effect is expected to appear in other cases, e.g., at another thickness of a dielectric layer if metal strips are coated by some dielectric of such a thickness that the total jump of phase of the electric field across the wall is the same both at the location of metal strips and at the bare dielectric layer.

\section{ACKNOWLEDGMENT}

The authors would like to thank A. I. Nosich for providing [13]. They would also like to thank the reviewers for helpful comments.

\section{REFERENCES}

[1] G. P. Tricoles, "Radome electromagnetic design," Y. T. Lo and S. W. Lee, Eds., in, Antenna Handbook. New York: Van Nostrand Reinhold, 1988, pp. 31-1-31-31.

[2] P. D. Einziger and L. B. Felsen, "Rigorous asymptotic analysis of transmission through a curved dielectric slab," IEEE Trans. Antennas Propagat., vol. AP-31, pp. 863-869, Nov. 1983.

[3] R. K. Gordon and R. Mittra, "Finite element analysis of axisymmetric radomes," IEEE Trans. Antennas Propagat., vol. 41, pp. 975-981, July 1993.

[4] V. B. Yurchenko, A. Altıntaş, and A. I. Nosich, "Numerical optimization of a cylindrical reflector-in-radome antenna system," IEEE Trans. Antennas Propagat., vol. 47, pp. 668-673, Apr. 1999.

[5] A. Altıntaş, A. Bircan, and V. B. Yurchenko, "Approximating circular radome by a dielectric slab in the antenna simulations," in $\mathrm{H}$. Groll and I. Nedkov, Eds., Microwave Physics and Techniques. London, U.K.: Kluwer, 1997, pp. 291-296.

[6] E. Bleszynski, M. Bleszynski, and T. Jaroszewicz, "Surface-integral equations for electromagnetic scattering from impenetrable and penetrable sheets," IEEE Antennas Propagat. Mag., vol. 35, pp. 14-25, Dec. 1993.

[7] R. C. Hall and R. Mittra, "Scattering from a periodic array of resistive strips," IEEE Trans. Antennas Propagat., vol. AP-33, pp. 1009-1011, Sept. 1985.

[8] R. Petit and G. Tayeb, "Theoretical and numerical study of gratings consisting of periodic arrays of thin and lossy strips," J. Opt. Soc. Amer., vol. 7, pt. A, no. 9, pp. 1686-1692, Sept. 1990.

[9] L. B. Felsen, "Complex-source point solutions for the field equations and their relation to the propagation and scattering of Gaussian beams," in Symposia Mathematica. New York: Academic, 1976, vol. 39, pp. $39-56$.

[10] I. V. Lindell, Methods for Electromagnetic Field Analysis. Oxford, U.K.: Clarendon, 1992, ch. 5.5, pp. 148-154.

[11] T. Oğuzer, A. Altıntaş, and A. I. Nosich, "Accurate simulation of reflector antennas by the complex source-Dual series approach," IEEE Trans. Antennas Propagat., vol. 43, pp. 793-801, Aug. 1995.

[12] K. M. Mitzner, "Effective boundary conditions for reflection and transmission by an absorbing shell of arbitrary shape," IEEE Trans. Antennas Propagat., vol. AP-16, pp. 706-712, May 1968.

[13] Ya. R. Grinberg, "Boundary conditions for electromagnetic field in the presence of thin metal membranes," Radiotekhnika i Elektronika [Sov. J. Commun. Technology and Electronics], vol. 26, pp. 2493-2499, Dec. 1981 (in Russian).

[14] T. B. A. Senior, "Combined resistive and conductive sheets," IEEE Trans. Antennas Propagat., vol. AP-33, pp. 577-579, May 1985. 
[15] T. B. A. Senior and J. L. Volakis, "Sheet simulation of a thin dielectric layer," Radio Sci., vol. 22, no. 7, pp. 1261-1272, Dec. 1987.

[16] A. I. Nosich, V. B. Yurchenko, and A. Altıntaş, "Numerically exact analysis of a two-dimensional variable-resistivity reflector fed by a complex-point source," IEEE Trans. Antennas Propagat., vol. 45, pp. 1592-1601, Nov. 1997.

[17] A. I. Nosich, Y. Okuno, and T. Shiraishi, "Scattering and absorption of $E$ - and $H$-polarized plane waves by a circularly curved resistive strip," Radio Sci., vol. 31, no. 6, pp. 1733-1742, Nov./Dec. 1996.

[18] R. F. Harrington and J. R. Mautz, "An impedance sheet approximation for thin dielectric shells," IEEE Trans. Antennas Propagat., vol. AP-23, pp. 531-534, July 1975.

[19] L. D. Landau and E. M. Lifshitz, Electrodynamics of Continuous Media. New York: Pergamon, 1984.

[20] G. Bouchitte and R. Petit, "On the concepts of a perfectly conducting material and of a perfectly conducting and infinitely thin screen," Radio Sci., vol. 24, no. 1, pp. 13-26, Jan./Feb. 1989.

[21] L. Li, "Use of Fourier series in the analysis of discontinuous periodic structures," J. Opt. Soc. Amer., vol. 13, pt. A, no. 9, pp. 1870-1876, Sept. 1996.

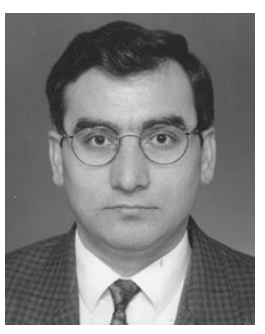

Ayhan Altıntaş (S'82-M'86-SM'93) was born on March 29, 1958, in Yozgat, Turkey. He received the B.S. and M.S. degrees from the Middle East Technical University (METU), Ankara, Turkey, in 1979 and 1981, respectively, and the Ph.D. degree from The Ohio State University, Columbus, $\mathrm{OH}$, in 1986, all in electrical engineering.

From 1981 to 1987, he was with the ElectroScience Laboratory of The Ohio State University. At present, he is a Professor at the Department of Electrical and Electronics Engineering, Bilkent University, Ankara, Turkey. He has held Research Fellowship and Guest Professor positions at Australian National University, Canberra, Tokyo Institute of Technology, Japan, and the Technical University of Munich, Germany. His research interests include electromagnetic radiation and scattering, microwaves, fiber optics, and integrated optics.

Dr. Altıntas is the recipient of the ElectroScience Laboratory Outstanding Dissertation Award of 1986, the IEEE 1991 Outstanding Student Branch Counselor Award, the 1991 Research Award of the Professor Mustafa N. Parlar Foundation of METU, and the 1996 Young Scientist Award of TUBITAK. He is a member of Sigma Xi and Phi Kappa Phi.
Slim Ouardani, photograph and biography not available at the time of publication.

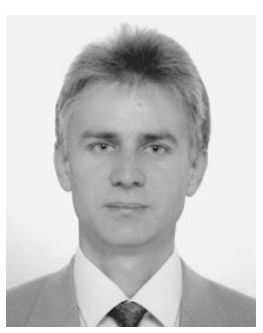

Vladimir B. Yurchenko was born in Kharkov, Ukraine, in 1957. He received the M.S. degree (with honors) from the Physics and Technology Department, Kharkov Polytechnic Institute, Kharkov, Ukraine, the Ph.D. degree in solid-state physics from the Kharkov Polytechnic Institute, and the D.Sc. degree from the Institute of Radiophysics and Electronics (IRE), Ukrainian Academy of Sciences, Kharkov, in 1980, 1984, and 1994, respectively.

From 1984 to 1995 , he was with the Department of Materials for Electronics and Solar Cells, Kharkov Polytechnic Institute, as a Research Scientist and an Associate Professor. His research interests there included the theory of hot-electron transport, photovoltaics, and electrical instability in semiconductors. Since 1995 he has been with the IRE as a Senior Research Scientist in the Department of Nonlinear Dynamics of Electronic Systems. Since 1996, he has been a member of the Visiting Faculty at the Department of Electrical and Electronics Engineering, Bilkent University, Ankara, Turkey. His current interests include computer simulation of electron transport in nanostructures, dynamical chaos in electronic devices, and wave scattering in electromagnetic systems. 